\title{
Los pactos sociales y el contrabando en la frontera colombo-venezolana
}

\section{Social Contracts and Contraband: A study in the Colombian Venezuelan Border}

Neida Albornoz-Arias (10) https://orcid.org/0000-0001-7851-5985

Universidad Simón Bolivar, Colombia,n.albornoz@unisimonbolivar.edu.co

Rina Mazuera-Arias (D) https://orcid.org/0000-0002-9888-5833

Universidad Simón Bolivar, Colombia, r.mazuera@unisimonbolivaredu.co

Ma Genoveva Millán-Vázquez de la Torre (1) https://orcid.org/0000-0002-6635-4456

Universidad Loyola Andalucia, España, gmillan@uloyola.es

Roberto Briceño-León (D) https://orcid.org/0000-0002-8882-7787

Universidad Central de Venezuela, Venezuela, roberto.bricenoleon@gmail.com

\begin{abstract}
Present-day social contracts and border contraband on the Northern Santander Department (Colombia) and Táchira State (Venezuela) are promoted by various actors. A dynamic economy that responds to a problem encouraged by the difference between the economies of both countries, generating asymmetry in the differential exchange, cost of goods and services, and forming part of the social, cultural and economic relations in the border under study; there are many families that sustain their economy with this income. Consequently, the article analyzes these relationships from a first focus, with a Categorical Regression analysis in Optimal Scaling (CatReg) over two samples: 2,394 and 1,398 people, respectively. Results were shown in following significant independent variables with more importance in both contexts: armed groups outside the law are part of the border dynamics and the perception of an environment of illegality, and the behavior of border people not based on conventional rules.
\end{abstract}

Key words: social contracts, space, territory, border, contraband.

Resumen: Los pactos sociales y el contrabando en la frontera Norte de Santander (Colombia)-Táchira (Venezuela) son promovidos por diversos actores. Esta dinámica responde a un problema alentado por la diferencia entre los modelos económicos de ambos países, que genera asimetrías en el diferencial cambiario y costo de bienes y servicios, creando parte de la dinámica social, cultural y económica de esta frontera. Son numerosas las familias que sostienen su economía con estos ingresos. Por ello, el artículo emprende un

Recepción:

$12 / 12 / 17$

Aprobación: $18 / 02 / 19$ primer análisis de estas relaciones con la aplicación de la Regresión Categórica, mediante el Escalamiento Óptimo (CatReg) en dos muestras de 2.394 y 1.398 personas. El resultado indicó que las variables independientes más significativas en ambos contextos son las siguientes: los grupos armados al margen de la ley son parte de la dinámica fronteriza, y la percepción de un ambiente de ilegalidad y comportamiento de las personas no basado en normas convencionales.

Palabras clave: pactos sociales, espacio, territorio, frontera, contrabando. 


\section{Introducción}

Las relaciones sociales y los pactos implícitos o explícitos que desarrollan los pobladores, o grupos de ellos, en un contexto geográfico, ocasionan que se fomenten e institucionalicen unas relaciones específicas de cooperación y reciprocidad. Estas motivan las acciones individuales de sus miembros, sin que exista una total conciencia de ello; no obstante, factores extrínsecos como el territorio y la cultura generan la aparición de cierto alineamiento social. Por ello resulta importante estudiar el espacio y territorialidad fronteriza para comprender esos pactos como constructo de sus habitantes, pero enfocados a partir de las acciones de contrabando.

Toda frontera tiene distintas concepciones que pueden relacionarse con los conceptos espacio y territorio; por tanto, se comienza revisando la noción de estos elementos. Con base en la naturaleza del objeto de estudio, en el presente trabajo se examinan dichos elementos desde una perspectiva de enfoque sociológico.

Concebido más allá de una relación de emplazamiento o ubicación desde la noción demográfica, donde existen relaciones de vecindad entre puntos o elementos, donde lo interesante es saber el tipo de relación y circulación, el espacio se entiende como el ámbito donde se hallan imágenes asociadas con relaciones grupales que se originan en distintas representaciones, y que dependen de los individuos, colectivos o de circunstancias. En este sentido, y según los criterios de Simmel (1977), todo espacio limitado, o con límites, produce una o unas reacciones psicológicas diferentes de aquellas que son generadas por los espacios no limitados, o con límites abiertos, pues en este concepto de espacio se conjuga el sitio físico y las dimensiones sociales, culturales, históricas, económicas y simbólicas. Un todo modificado por la historia y donde lo más relevante son las relaciones de enlace e interacción de los integrantes del espacio.

Otros autores, como Vargas (2012: 321), sostienen que aquellas relaciones que se producen en los espacios donde se involucran actividades humanas como las: agrarias, urbanas, topográficas, biogeográficas, de servicios, de hábitats, entre otras, se dan sobre estructuradas organizaciones espaciales de extensión variada, las cuales comprenden escalas con magnitudes que van desde el nivel local hasta el mundial. Allí cada espacio organizado se conecta con otros a través de flujos de energía y materiales.

Al pasar a la dimensión territorio, Raffestin (1986) señala que este se encuentra en el centro de las representaciones de la complejidad que nos rodea. Resulta así, en primer lugar, como espacio geográfico, pero esto podría 
limitarse solo al medio físico, es decir, al que rodea y sustenta a una realidad humana. Luego, el término de territorio es una definición más amplia que el de espacio físico, porque combina el medio físico natural y el ordenado o humanizado, abarcando a las personas que se apropian de él. Estas premisas permiten dilucidar la confusión que se puede presentar al señalar al espacio y territorio como una sola expresión.

En consecuencia, el territorio es el espacio elegido simbólica e instrumentalmente y ocupado por los actores sociales (individuales y colectivos), y considerado por igual como un área estratégica geopolíticamente o como jurisdicción político-administrativa (Bonnemaison, 2001; Sack, 1997).

Desde el punto de vista de Brenna (s.f.) y Geiger (1994), también se puede entender el territorio como un espacio o extensión terrestre delimitado, donde se propician relaciones de poder, de gestión y de control del Estado, de individuos, grupos u organizaciones, y que son expresadas como territorialidad. De allí se desprende un vínculo de afectividad, identidad y lealtad por parte de los actores territoriales, los cuales, si bien tienen intereses, percepciones, valores y actitudes distintas, pueden generar relaciones de colaboración y reciprocidad o de conflicto.

Según Quijada (2000), el territorio también es utilizado como elemento cultural e ideológico desde el nacionalismo, como elemento de cohesión social y como marco económico y físico, donde se cimientan los mercados y se despliegan las estrategias geopolíticas.

La frontera también se presenta como un espacio geográfico en el cual la definición de territorio complementa la complejidad y dinámicas que le caracterizan, siendo para Quijada (2000) donde se establece el control estatal tanto del espacio como de la producción de la identidad nacional. Por frontera se ha considerado a la región que separa a dos o más países y comprende dos elementos: límite y borde.

El límite es la línea divisoria, y el borde es la zona contigua al límite. No obstante, la frontera transciende los aspectos del límite, por ser un área de integración y/o separación; una zona de transición entre los territorios, donde existen e interactúan diferentes normas, pactos o acuerdos que identifican a cada territorio. Ahí, el límite representa una barrera y una membrana permeable como lo definieran y explicaran Sánchez (2015) y Taylor y Lawrence (2007). Por ello, parte de las dinámicas sociales, económicas y culturales que identifican a la frontera no pueden ser enmarcadas dentro del espacio geográfico o territorio que las describen, en virtud del impacto que generan más allá de los límites que la identifican. 
Desde este contexto teórico, el objetivo del presente artículo se centró en el estudio de los pactos sociales y las actividades de contrabando que se dan en la frontera formada por los límites orientales del Departamento Norte de Santander, en Colombia, con el límite occidental del Estado Táchira, en Venezuela. Para ello, se empleó el método de Regresión Categórica con Escalamiento Óptimo (CatReg), considerando 12 variables categóricas (algunas ordinales y otras nominales) y la variable dependiente: visualización de pactos sociales informales (acuerdos entre personas y/o instituciones) en la zona de frontera (categórica ordinal).

La herramienta estadística o software SPSS permitió describir, cuantificar y determinar las relaciones entre las variables predictoras y la variable dependiente. Asimismo, se analizó la relación entre los pactos sociales y el contrabando, debido a que este último forma parte de la dinámica social, cultural y económica en la frontera referida. Un elevado número de familias sostienen su economía con los ingresos del contrabando; de ahí el interés de los investigadores por conocer la percepción de los encuestados sobre estos temas. Por último, los resultados estadísticos del estudio se presentan en tres tablas al final del artículo.

Si bien el contrabando en la frontera Norte de Santander (Colombia)Estado Táchira (Venezuela) es facilitado y promovido por diversos actores, esta dinámica económica se da, con mayor intensidad, a partir de la quiebra de la economía de Venezuela, desde 2012, robustecida por la diferencia entre los modelos económicos de ambos países, los cuales generan asimetrías en el diferencial cambiario, costo de bienes y servicios, entre otras. Coincidiendo con Tejedor (2012), quien define a Colombia como un país con un modelo económico orientado al capitalismo y libre mercado, se contrapone Venezuela con un modelo económico de índole socialista-comunista, con controles estatales de cambio de divisas, de los medios de producción y de precios.

Según Álvarez (2007), esto ha perjudicado la producción nacional, promoviendo la importación de bienes, al ser acompañada de una política de subsidios populistas en alimentos de la canasta básica, medicamentos y combustibles. En la actualidad, el quiebre económico venezolano ha ocasionado una crisis política y social, donde predomina la inflación y escasez, originando una situación que continúa potenciando la práctica del contrabando, a pesar de los esfuerzos de las autoridades de ambos lados de la frontera por controlarlo. 


\section{Una de las más complejas y dinámicas fronteras internacionales en Latinoamérica}

Para Rhi-Sausi et al. (2009), el establecimiento de Estados nacionales en América Latina propició la existencia de 36 fronteras a lo largo de 41.000 kilómetros. Las mismas son concebidas como espacios "interestatales", donde convergen relaciones entre sus habitantes con las distintas instancias del Estado y donde las competencias deben ser compartidas en el ámbito de gobiernos regionales y locales; mientras que lo concerniente a la línea fronteriza, queda reducido a solo un asunto de los gobiernos nacionales de cada país.

Sostienen por igual Rhi-Sausi et al. (2009) que cada espacio fronterizo posee características propias que le permiten construir, a su vez, una tipología propia. Es decir, cada frontera es una realidad única, y la frontera occidental entre Colombia y Venezuela no es la excepción. Allí se cruzan "universos simbólicos diferentes y desiguales" que hacen vida en ella y participan en una economía fronteriza, la cual se diversifica entre lo legal e ilegal y donde intervienen "actores transfronterizos" (Carrión y Espín, 2011: 13). Entre otras problemáticas, en esta región se da el contrabando como una práctica comercial recurrente, promovida por distorsiones en el mercado, debido a la coexistencia de economías y relaciones jurídicas dispares, que generan acentuadas diferencias.

La totalidad de la frontera entre Colombia y Venezuela comprende una extensión de 2.219 kilómetros, y es la más larga de las fronteras colombianas. Desde el norte hasta el sur comprende a los departamentos de La Guajira, Cesar, Norte de Santander, Boyacá, Arauca, Vichada y Guainía, y por el lado venezolano a los estados Zulia, Táchira, Apure y Amazonas. Autores como Carreño (2014) señalan que el tráfico de estupefacientes y el contrabando de gasolina, alimentos de la canasta básica, medicamentos, productos agroindustriales, entre otros, son los mayores negocios ilícitos que identifican a esta frontera.

En la región nororiental de Colombia se encuentra el Departamento Norte de Santander, el cual limita con el Estado Táchira (en Venezuela). Aquí, el comercio legal e ilegal con las ciudades fronterizas del Táchira como San Antonio, Ureña y San Cristóbal han sido históricamente la principal fuente de ingresos del departamento. Las dos primeras ciudades bordean la frontera y la tercera es la capital del Estado Táchira, la cual se conecta a dicha frontera por diferentes vías terrestres.

Sobre las relaciones socioespaciales en esta región, Carreño (2014) explica que desde la época colonial española siempre ha existido un flujo 
migratorio de personas (colombianos y venezolanos) de un lado hacia el otro, siendo una de las razones la económica. Esto ha originado una dinámica que se mueve entre las acciones legales e ilegales.

Dentro de las acciones ilegales se cuentan los delitos transnacionales, por ejemplo, lavado de dinero, tráfico de drogas, de personas, armas, de órganos y de tecnologías, entre otros. Como lo describen Niño et al. (2012), otra muestra de esta dinámica incluye la presencia de grupos organizados al margen de la ley, que operan mediante una acción concertada, utilizando violencia, corrupción u otros medios, para conseguir directa o indirectamente altos beneficios económicos, entre los cuales se encuentra el contrabando.

En este tipo de contrabando pueden distinguirse dos modalidades: 1) el paso de pequeñas cantidades y 2) el de grandes cantidades de mercancías que no pagan aranceles o impuestos. En el caso de las pequeñas cantidades, estas se concentran en el tráfico de alimentos y productos de consumo masivo, debido al diferencial en el tipo de cambio entre la moneda de Colombia y de Venezuela. Respecto al paso de grandes cantidades, está vinculado a grupos de carácter guerrillero, paramilitares, organizaciones criminales o a la delincuencia común, que se enfrentan por el control de los caminos o rutas informales para el tránsito de mercancías, y quienes "ostentan el monopolio así como las ganancias que se concentran en pocas manos" (Albornoz et al., 2016: 101).

Estos individuos y grupos actúan en complicidad con funcionarios o cuerpos de seguridad venezolanos que evidencian un comportamiento de “cooperación cooperativa”, en términos de Traxler y Spichtig (2011), y entre funcionarios públicos y contrabandistas en la frontera Norte de Santander y Táchira, caracterizando profundamente a esta dinámica socioespacial.

Los grupos paramilitares se originaron en Colombia en 1990, bajo la denominación de "Autodefensas Unidas de Colombia" (AUC), con el objetivo de combatir la guerrilla. Los paramilitares se apropiaban de los territorios para obtener beneficios económicos que les permitieran seguir ejecutando sus prácticas criminales. Parte de ellos, los conocidos por los calificativos de "urabeños" y "rastrojos", operan en la frontera colombo-venezolana y se sustentan de beneficios del narcotráfico, el contrabando y la extorsión (BBC Mundo, 2015; Carreño, 2014; Niño et al., 2012).

De igual modo, están inmersos en el negocio del contrabando grupos guerrilleros, principalmente del Ejército de Liberación Nacional (ELN) y del Ejército Popular de Liberación (EPL), que existen en Colombia desde el conflicto interno iniciado en el siglo pasado y cuyas manifestaciones de violencia han afectado a todo el país (Niño et al., 2012; Rodríguez-Albor et al., 2016). 


\section{El pacto social y su teoría como un instrumento eficaz para enfrentar el problema del contrabando en América Latina y el Caribe}

Con el desarrollo de la humanidad, las sociedades han requerido instaurar normas y leyes que les permitan regular sus relaciones sociales, fijar responsabilidades e instaurar mecanismos que censuren o reprochen conductas ajenas a dicha relación. A tal efecto, el Estado erige reglas que hacen predecible el comportamiento de cada uno de los actores en la sociedad, lo cual, bajo una perspectiva de estabilidad, crea pactos para que dichas relaciones se den en armonía, a través del establecimiento de normas asentadas, en principio, en la Constitución política de dicho Estado. Son convenios que, según Bobbio (2005), se instituyen como acuerdos jurídicos entre individuos que buscan una convivencia pacífica.

Para Avdagic (2011), estos pactos han sido instrumentos para diseñar políticas efectivas de control social, regulación y comportamiento ciudadano. De igual manera, se han utilizado como herramientas para implicar a los grupos representativos de la sociedad en las reformas sociales (Habermas, 1989). Por ello, dado el alcance de esta noción de compromiso, autores como Daros (2005) señalan que el pacto social exige el mutuo respeto de las personas que lo hacen, y representa normas de respeto mutuo a la vida en libertad, simbolizando el intercambio político continuo entre los actores sociales de manera autónoma. Son normas que, según Öberg et al. (2011), desarrollan interacciones.

Dichas normas son conocidas como instituciones, reglas de juego que generan cambio social (Drobak y Nye, 1997; North, 1994). Igualmente se refieren a las condiciones establecidas e impuestas por las personas que propician la interacción social, económica y política, según los términos expuestos por Briceño-León (2016). Tales reglas pueden ser formales, como las establecidas en las Constituciones y leyes legalmente reconocidas y codificadas para el conocimiento público; o informales, que se encuentran en las costumbres, tradiciones y normas sociales (Coleman, 1994; Prats, 2007).

En cuanto a las normas formales, Posada et al. (2015) explican que estas funcionan como leyes que regulan la conducta entre los particulares, estableciendo lo que puede y no puede hacerse. Por su parte, las normas informales (sociales) se definen como los valores comunes de un grupo que influyen en el comportamiento de un individuo, a través de la internalización de las preferencias de una sociedad (Mixon et al., 2015).

Del mismo modo, al concebir a una norma social como una regularidad, Plancarte (2015) señala que la misma puede emerger a partir de un proceso 
donde cierta práctica o acción comienza a realizarse de forma periódica. Es decir, se codifica a partir de que un individuo o grupo empieza a dejar establecida cierta conducta, en un contexto donde otras personas estarán dispuestas a igualar tal actitud. Este proceso se concretiza muchas veces por inercia o por creer que esa práctica tiene un sentido. Por ello, culturalmente, dicha práctica se hace periódica; no realizarla es "mal visto", máxime cuando la sanción aparece. Esto implica que tal práctica se convierta en una norma.

Desde la perspectiva del funcionalismo estructural de Parsons (1997), los sujetos sociales crean sistemas de interacción: cultural (pautas de conducta, símbolos, creencias), de personalidad (apropiación individual de las normas), social (división del trabajo y estratificación social). Por lo tanto, se asume que las normas sociales se determinan por la cultura y afectan las motivaciones individuales, siendo que algunos pueden incumplirlas; aunque la sociedad tipificará la conducta de esas personas como inconcebible, lo cual les obliga a corregirla (Collins, 1996). De acuerdo con Elster (1989: 16), las normas sociales difieren de las normas morales y jurídicas.

El término normas sociales implica una categoría de acción que incluye órdenes, prohibiciones y permisos, que se pueden diferenciar de otros tipos de consideraciones prácticas "ontológicas, epistemológicas y bases normativas" (Morrow, 2014). Existen cuatro características claves de las normas sociales que indican su carácter distintivo: 1) la función de la particularidad, 2) la práctica-arraigo, 3) la función de grupo intencional y 4) la función de rendición de cuentas de su creación.

Según la particularidad y práctica-arraigo, las normas sociales circulan dentro de los grupos específicos, organizaciones o sociedades, y son normativas solo para los miembros de estos. Sin embargo, para Zorrilla (1992) el exceso de normas en una sociedad fomenta o incrementa la corrupción como mera violación de la legalidad. La violación de la norma o también llamada conducta desviada es una expresión de los fenómenos de homeostasis social, tal como ocurre en la mayor parte de los casos con el denominado mercado negro, informal o marginal. Para Calhoun et al. (2000), dichas desviaciones son el resultado de la interacción de la conducta individual, los problemas sociales y las condiciones sociales.

Por ende, los pactos surgen de acuerdos y se materializan a través de normas legales, morales o sociales. Los pactos sociales se plasman mediante normas sociales informales, ya sea legales o ilegales. Calderón (1999) considera que la emergencia de pactos sociales y, en especial los ilegales, se presenta no solo por las diferentes problemáticas que enfrentan los individuos, sino también se sustenta en acciones de ingobernabilidad y problemas 
del mercado, que conllevan a las personas a crear acuerdos paralelos con los cuales satisfagan de algún modo sus necesidades.

Los pactos sociales pueden ser algo positivo para la sociedad cuando no incluyen la ilegalidad. Carmona (2006) sostiene que para los entes gubernamentales y las instituciones sociales en general estos pactos pueden llegar a ser una modalidad del desarrollo local, mediante el trabajo colectivo y la negociación, por lo cual podrían ser incorporados como elemento de refuerzo en la lucha contra el contrabando en las fronteras de América Latina y el Caribe.

\section{Un caso específico de pactos sociales y contrabando en la frontera colombo-venezolana}

\section{Metodología y procesamiento de datos}

La investigación, desde un estudio de carácter exploratorio-descriptivo, obtuvo y analizó los datos a partir de una encuesta semiestructurada, aplicada a personas (jefes de hogar) de entre 18 y 69 años. La población para el Departamento Norte de Santander (Colombia) estuvo constituida por $\mathrm{N}=830.872$ habitantes (DANE, 2015); el tamaño de la muestra correspondió a $n=2.394$, un nivel de confianza de 95\%, y error máximo admisible de $2 \%$. El tipo de muestreo fue estratificado, con afijación proporcional al tamaño de la población de las áreas geográficas del departamento. En el Estado Táchira (Venezuela), la población correspondió a $\mathrm{N}=317.594$ habitantes (INE, 2012); el tamaño de la muestra a $n=1.398$, un nivel de confianza de $95 \%$, y error máximo admisible de 3\%.

Para garantizar la representatividad de la muestra se utilizó como marco muestra el registro de unidades inmobiliarias en ambos contextos. Las características de dicha muestra se pueden observar en la Tabla 1.

Asimismo, se observó la conformación de pactos sociales informales (acuerdos entre personas y/o instituciones) en la zona de la frontera, y dicha acción quedó definida como variable dependiente. Esta fue seleccionada al considerar que una de las características de las fronteras entre países en América Latina son las marcadas y diversas relaciones económicas, sociales y culturales -entre ellas, crímenes y violencia-, las cuales, según Carrión (2011), nacen y se propagan a partir de factores sociopolíticos, de asimetrías económicas y legales, de aspectos institucionales y ético-culturales. 


\section{Caracterización de las variables}

En el presente estudio se consideraron 12 variables independientes: 1) género, 2) edad, 3) nivel educativo, 4) condición de ocupación, 5) existencia de relaciones de cooperación entre los funcionarios de seguridad y los practicantes del contrabando, 6) la burocracia en los puestos de seguridad para controlar la mercancía de contrabando ha aumentado el pago de sobornos, 7) el Estado colombiano actúa de manera eficaz en el control de la mercancía de contrabando, 8) hay confianza en las instituciones del poder público para limitar el flagelo del contrabando, 9) los grupos al margen de la ley son parte de la dinámica fronteriza, 10) grandes cantidades de mercancía de contrabando que se trafican gracias a las relaciones de cooperación con los funcionarios y los comerciantes informales, 11) se percibe un ambiente de ilegalidad, y 12) el comportamiento de las personas no se basa en normas en la frontera; el gobierno nacional y regional ha notificado y publicado la información acerca de los hallazgos, leyes, decretos y resoluciones relacionadas al contrabando.

Con la finalidad de describir, cuantificar y determinar las relaciones entre las variables predictoras y la variable dependiente, determinando la influencia o importancia de dichas relaciones sobre la variable dependiente, dada la naturaleza de evaluación cualitativa de las mismas, se aplicó la técnica estadística de análisis multivariante conocida como Regresión Categórica con Escalamiento Óptimo o CatReg (De Leeuw, 2005; IBM Knowledge Center, s.f.; Preisser y Koch, 1997; Van Der Kooij y Wetenschappen, 2007).

\section{Análisis empirico}

En el modelo inicial se incluyeron todas las variables y se obtuvieron las contribuciones parciales a la explicación de la varianza de todos los coeficientes estandarizados de las variables transformadas, así como también el nivel de significación en el modelo, teniendo como resultado para el Norte de Santander un $\mathrm{R}^{2}-.397$ y $(\mathrm{F}=72.587, p<.05)$, y cuatro variables no significativas: 1) sexo, 2) la burocracia en los puestos de seguridad para controlar la mercancía de contrabando ha aumentado el pago de sobornos, 3) el Estado colombiano actúa de manera eficaz en el control de la mercancía de contrabando y 4) hay confianza en las instituciones del poder público para limitar el flagelo del contrabando.

En el caso del Táchira, el resultado fue un $\mathrm{R}^{2}-.517$ y $(\mathrm{F}=69.003, p<.05)$, no aportando significancia cinco variables: 1) sexo, 2) edad, 3) existen relaciones de cooperación entre los funcionarios de seguridad y los practicantes 
del contrabando, 4) hay confianza en las instituciones del poder público para limitar el flagelo del contrabando, y 5) el gobierno nacional y regional ha notificado y publicado la información acerca de los hallazgos, leyes, decretos y resoluciones relacionadas al contrabando.

Para optimizar el modelo, se excluyeron secuencialmente las variables que no aportan significancia (bajo el criterio de pasos sucesivos). Este proceso se realizó de forma sistemática hasta obtener, en conjunto, todas las variables significativas que explican el mejor ajuste.

\section{Validez del modelo y resultados}

El resultado del análisis de la varianza (ANOVA) presenta la validez del modelo. En el caso del Norte de Santander, el estimador de la varianza de la regresión (63.521) es mayor que el estimador de la varianza del residuo (.606) y el valor del estadístico de contraste y el nivel crítico asociado ( $\mathrm{F}=$ $104.813 ; p=.000)$. Esto indicó que el ajuste es estadísticamente significativo $(\mathrm{p}<.05)$. En el modelo del Táchira, el estimador de la varianza de la regresión (48.702) fue mayor que el estimador de la varianza del residuo (.483) y el valor del estadístico de contraste y el nivel crítico asociado ( $\mathrm{F}=$ $100.840 ; p=.000)$, mostrando que el ajuste resultó estadísticamente significativo $(\mathrm{p}<.05)$. El valor del coeficiente de determinación ajustado en el caso del Norte de Santander es $\mathrm{R}^{2}-.394$ y en el Táchira, $\mathrm{R}^{2}-.517$; esto indica que $39.4 \%$ y $51.7 \%$, respectivamente, de la variabilidad de la variable dependiente está explicada por las variables predictoras consideradas en el modelo óptimo.

En la Tabla 2 se presentan los coeficientes de regresión parcial estandarizados de las variables transformadas asociadas al modelo. Todos ellos muestran aportes significativos a los valores de la variable modelo $(p<.05)$, señalando el cambio de las cuantificaciones que cada variable predictora produce en la variable dependiente.

Tanto en el Norte de Santander como en el Táchira, los dos coeficientes estandarizados de las variables transformadas predictoras de mayor aporte de correlación al modelo son: 1) los grupos al margen de la ley son parte de la dinámica fronteriza, 2) se percibe un ambiente de ilegalidad y el comportamiento de las personas no se basa en normas en la frontera (véase Tabla 2). 


\section{Diagnóstico a partir de los resultados}

$\mathrm{Al}$ implicar los principios teóricos de los pactos sociales como: el respeto mutuo entre las personas, los compromisos de quienes pactan y el intercambio entre los actores que hacen vida en el territorio, queda evidenciado que en la frontera Norte de Santander-Táchira existen pactos sociales. De forma particular, se detectaron normas sociales impuestas por los actores de la sociedad, las cuales funcionan para la convivencia, aceptación e intervención en conductas ilegales.

Se trata de la existencia de pactos o acuerdos informales (y en este caso ilegales) relacionados con el contrabando, así como acuerdos entre los actores, donde los grupos armados ilegales -que hacen presencia en la zonaintervienen en este negocio, traficando las mercancías de un país a otro y controlando las zonas grises de paso fronterizo, conocidas como trochas (Carrión, 2011; Niño et al., 2012; Stein et al., 2015).

También se observó la influencia negativa sobre la variable dependiente de algunas variables predictoras (véase Tabla 2); en el Norte de Santander: 1) nivel educativo, 2) condición de ocupación, 3) el gobierno nacional y regional ha notificado y publicado la información acerca de los hallazgos, leyes, decretos y resoluciones relacionadas al contrabando. En el caso del Táchira: 1) la burocracia en los puestos de seguridad para controlar la mercancía de contrabando ha aumentado el pago de sobornos.

Lo anterior indica una percepción negativa, pues las personas consultadas en el Norte de Santander -indistintamente de su nivel educativo y condición laboral- consideran que hay pactos sociales informales en la zona (acuerdos entre personas e instituciones). Asimismo, opinan que el gobierno nacional y regional no ha notificado y publicado la información acerca de los hallazgos, leyes, decretos y resoluciones relacionadas al contrabando.

Esto refuerza lo expuesto por Niño et al. (2012), quienes explican cómo en los pactos establecidos entre los distintos actores que facilitan y promueven el contrabando en la frontera Norte de Santander-Táchira se encuentran autoridades militares y policiales, comerciantes, grupos armados al margen de la ley y quienes controlan las zonas grises por donde transitan las mercancías y los propios contrabandistas. Estos pactos son difíciles de controlar, debido a la impunidad en la práctica del contrabando, motivo por el cual este negocio permea la cultura y conlleva a la tácita aceptación social.

En el Táchira, la percepción negativa de los encuestados queda en evidencia al considerar que la existencia de pactos sociales informales en la zona (acuerdos entre personas e instituciones) no es producto de la burocracia en 
los puestos de seguridad para controlar la mercancía de contrabando y el aumento del pago de sobornos (véase Tabla 2).

Lo anterior coincide con Castañeda (2015), quien señala que la percepción sobre la corrupción en una región está relacionada con el imaginario común que se crean las personas sobre el funcionamiento del Estado y sus instituciones, y no necesariamente con la experiencia o vivencia que se tenga con este problema. Stein et al. (2015) refieren, por igual, que el contrabando representa una red de corrupción que permea todas las esferas de los actores involucrados.

Respecto a las medidas de importancia relativa de las variables predictoras, para la variable dependiente se observó que en el Norte de Santander los siguientes predictores: 1) los grupos al margen de la ley son parte de la dinámica fronteriza, 2) se percibe un ambiente de ilegalidad y el comportamiento de las personas no se basa en normas en la frontera, y 3) existen relaciones de cooperación entre los funcionarios de seguridad y los practicantes del contrabando, suman .93, es decir, representan el $93 \%$ de la relevancia (véase Tabla 3 ).

Este último (imaginario de relaciones de cooperación entre funcionarios de seguridad y contrabandistas) coincide con lo ya expuesto por Arancibia et al. (2013), al señalar que los miembros de la sociedad comparten representaciones sociales, pero algunos de ellos tienen experiencias, intereses y necesidades que los acercan más al fenómeno que perciben, como es el caso de los pactos sociales frente al contrabando.

Dicha situación también influye en el ejercicio del buen gobierno, pues la existencia de una marcada corrupción genera por igual una amplia desconfianza entre los habitantes de la zona hacia todo lo relacionado con el sector público (Albornoz y Mazuera, 2015).

Para el Estado Táchira, el conjunto de predictores: 1) los grupos al margen de la ley son parte de la dinámica fronteriza, y 2) se percibe un ambiente de ilegalidad y el comportamiento de las personas no se basa en normas en la frontera, suman .97 , es decir, representan $97 \%$ de la importancia (véase Tabla 3).

Cabe destacar que la percepción coincidente de los habitantes del Norte de Santander y del Táchira acerca de la presencia de grupos armados al margen de la ley como parte de la dinámica fronteriza, fue el predictor más importante, lo cual concuerda con Alba et al. (2016), quienes manifiestan que el contrabando -al ser una actividad lucrativa ilegal- requiere un control de territorios, a lo cual el Estado es indiferente, y está relacionada con la corrupción o complicidad entre lo público y privado, y la presencia de organizaciones 
mafiosas que se encargan de articular la economía legal e ilegal, financiando organizaciones legales y manteniendo el control de territorios y zonas de circulación de mercancías.

\section{Conclusión}

El imaginario de los pactos sociales informales (acuerdos entre personas y/o instituciones), en la zona de la frontera, que promueven y facilitan el contrabando, se plasmó en un estudio diagnóstico objetivo, a través de una encuesta de percepción aplicada a la muestra de habitantes del Departamento Norte de Santander (Colombia) y del Estado Táchira (Venezuela). Dicha encuesta se elaboró con la intención de evidenciar la percepción de la existencia de pactos sociales y sus causas, según las creencias de los ciudadanos. Todo ello, entendido como un aspecto clave para conocer la situación en cada país, conforme lo explican Villoria y Jiménez (2012).

Se alcanzaron dos modelos de regresión que cumplen con todos los supuestos y, por lo tanto, pueden ser útiles para visualizar las variables que influyen sobre la opinión de la percepción de la existencia de pactos sociales informales (acuerdos entre personas y/o instituciones) en la zona de la frontera (variable dependiente).

Estos modelos de regresión dejaron en evidencia que las variables predictoras que explican la variable dependiente, tanto en el contexto del Norte de Santander como en el del Táchira, son: 1) los grupos al margen de la ley son parte de la dinámica fronteriza, y 2) se percibe un ambiente de ilegalidad y el comportamiento de las personas no se basa en normas en la frontera.

Un hallazgo coincidente en el norte de Santander y en el Táchira fue identificar las variables con mayor importancia y comunes en ambos modelos. Estas fueron: 1) la presencia de grupos al margen de la ley son parte de las dinámicas en la frontera, y 2) la percepción de un ambiente de ilegalidad y el comportamiento de las personas no se basa en normas en la frontera. Es decir, la actividad ilegal del contrabando está ligada a grupos armados al margen de la ley, lo cual coincide con el concepto de anomia referido por Zorrilla (1992), como una conducta desviada de los actores sociales en la frontera Norte de Santander-Táchira, quienes a pesar de conocer las normas (anomia subjetiva), las han internalizado superficialmente y, además, las violan impunemente.

Por ello, toda percepción de pactos sociales informales o acuerdos entre personas $y / o$ instituciones relacionados con el contrabando en la frontera forma parte de una dinámica social que involucra a la población, ocasionando 
que se perciban dichos pactos. Las personas conocen las actividades ilícitas, actores, riesgos y consecuencias, y algunos jóvenes, como lo indica González (2016), ven en estas actividades un ejemplo a seguir.

En conjunto, esto permite reflexionar sobre las representaciones sociales, las cuales son constructos que se comparten entre los miembros de la sociedad. Es importante acotar que algunos de estos constructos son más cercanos a determinadas experiencias, por los intereses, necesidades o expectativas que tengan las personas frente al fenómeno que están percibiendo (Arancibia et al., 2013). Es el caso de los pactos sociales frente al contrabando, en los cuales puede observarse que todos los ciudadanos de la frontera son sensibles al hecho y a los pactos que giran en torno a este; razón por la cual esta investigación puede servir como punto de partida para otros trabajos, donde se profundice sobre esta realidad fronteriza latinoamericana.

Para el caso del Estado Táchira, dado que en su espacio resulta común encontrar productos circulando de manera ilegal en las calles, noticias sobre contrabando e involucrados en la práctica, vecinos y conocidos que hacen parte activa del ilícito, se ha creado una relación directa frente a esta percepción, donde la coyuntura política, el desabastecimiento, las formas de operar de los contrabandistas, las dinámicas sociales y familiares para conseguir los recursos básicos -así sean de contrabando-, han hecho que los pactos sociales ilegales fronterizos sean percibidos de manera más contundente o clara.

$\mathrm{Si}$ bien existe un límite que separa ambas naciones, Colombia y Venezuela, hay por igual un espacio prolongado donde confluyen dinámicas sociales, culturales, económicas (legales e ilegales). En él concurren dos legalidades o pactos sociales diferenciados: los formales de ambos países y los informales o ilegales de cada lado, que pueden ser iguales o distintos.

Por la problemática que se vive en la frontera del oriente colombiano y del occidente venezolano, es necesaria y perentoria la cooperación transfronteriza. Esta representaría una ayuda para las instituciones políticas, sociales y económicas legales, al evitar el desorden y la duplicidad de los esfuerzos, partiendo de la formulación de acertadas políticas de gobierno, acordes con la realidad local y regional, y de la definición de objetivos, establecimiento de funciones y propuestas de servicios entre ambas entidades, con base en lo expuesto por Rhi-Sausi et al. (2009).

En este contexto, la acción de los gobiernos locales juega un papel fundamental por su cercanía con el ciudadano, motivo por el cual es necesario fortalecer las capacidades de gestión de estos actores locales y la productiva articulación universidad-municipio en un todo integral. Ello resulta un verdadero desafío, a mediano plazo, para lograr la conformación de un amplio 
espacio de aprendizaje, con la creación de mecanismos que demanda toda integración fronteriza, según lo planteado por Oddone et al. (2016).

\section{Referencias}

Alba, Magaly et al. (2016), Percepción de los habitantes de las subregiones del Norte de Santander frente al contrabando, Colombia: Universidad Simón Bolívar.

Albornoz, Neida et al. (2016), Análisis del contrabando en el Norte de Santander, desde la percepción de los constructos de la institucionalidad, Colombia: Universidad Simón Bolívar.

Albornoz, Neida y Mazuera, Rina (2016), "Percepción de habitantes del Norte de Santander sobre limitaciones del buen gobierno”, en Revista Venezolana de Gerencia, vol. 21, núm. 75, Venezuela: Universidad del Zulia.

Álvarez, Ángel (2007), "Venezuela 2007: los motores del socialismo se alimentan con petróleo”, en Revista de Ciencia Politica, vol. 27, Chile: Pontificia Universidad Católica de Chile.

Arancibia, Susana et al. (2013), "Representaciones sociales en torno a equidad, acceso y adaptación en educación universitaria”, en Psicoperspectivas. Individuo y Sociedad, vol. 12, núm. 1, Chile: Pontificia Universidad Católica de Valparaíso. DOI: 10.5027/psicoperspectivas-Vol12-Issue1-fulltext-236 Disponible en: 10.5027/ psicoperspectivas-Vol12-Issue1-fulltext-236 [20 de agosto de 2016].

Avdagic, Sabina et al. (2011), "The conditions for pact. A fuzzy set analysis of the resurgence of Tripartite Concertation”, en Avdagic, Sabina et al. [comps.], Social Pacts in Europe: Emergence, Evolution, and Institutionalization, Estados Unidos: Oxford University Press.

BBC Mundo (British Broadcasting Corporation Mundo) (2015), "Crisis fronteriza entre Colombia y Venezuela: ¿Quiénes son los paramilitares de los que habla Nicolás Maduro?", en $B B C$ Mundo. Disponible en: http://www.bbc.com/mundo/ noticias/2015/09/150908_venezuela_colombia_paramilitares_maduro_dp [12 de octubre de 2016].

Bobbio, Norberto (2005), Liberalism and Democracy, Estados Unidos: Verso.

Bonnemaison, Jöel (2001), La géographie culturelle. Cours de l'Université Paris IV-Sorbonne, 1994-1997, Francia: CTHS Edition.

Brenna, Jorge (s.f.), Espacio y Territorio: una mirada sociológica. Disponible en http:// slideflix.net/doc/163291/espacio-y-territorio--una-mirada-sociol\%C3\%B3gica-laintenci\%C3\%B3n-de [22 de agosto de 2017].

Briceño-León, Roberto (2016), Ciudades de vida y muerte: la ciudad y el pacto social para la contención de la violencia, Venezuela: Alfa.

Calderón, Julio (1999), "Algunas consideraciones sobre los mercados ilegales e informales de suelo urbano en América Latina”, en Reporte de Investigación LP99Z16. Disponible en: http://www.institutodeestudiosurbanos.info/dmdocuments/cendocieu/ Especializacion_Mercados/Documentos_Cursos/Algunas_Consideraciones_SobreCalderon_Julio-1999.pdf [16 de agosto de 2017].

Calhoun, Craig et al. (2000), Sociología, España: McGraw Hill.

Carmona, Rodrigo (2006), "Los pactos territoriales en Italia: Un análisis de la experiencia 
Neida Albornoz-Arias, Rina Mazuera-Arias, Ma Genoveva Millán-Vázquez de la Torre y Roberto Briceño-León. Los pactos sociales y el contrabando en la frontera colombo-venezolana

reciente y sus implicancias en términos de 'governance', en EURE, vol. XXXII, núm. 96, Chile: Pontificia Universidad Católica de Chile. DOI: 10.4067/ S0250-71612006000200007 Disponible en: http://dx.doi.org/10.4067/S025071612006000200007 [16 de agosto de 2017].

Carreño, Ángela María (2014), "Refugiados colombianos en Venezuela: Quince años en búsqueda de protección”, en Memorias: Revista Digital de Historia y Arqueología desde el Caribe, núm. 24, Colombia: Universidad del Norte. DOI: 10.14482/memor.24.6299 Disponible en: http://dx.doi.org/10.14482/memor.24.6299 [12 de agosto de 2017].

Carrión, Fernando (2011), "En el límite de la vida: La violencia fronteriza”, en Carrión, Fernando y Johanna, Espín [comps.], Relaciones fronterizas: Encuentros y conflictos, Ecuador: Facultad Latinoamericana de Ciencias Sociales.

Carrión, Fernando y Espín, Johanna (2011), "La geografía del delito en la lógica de la frontera”, en Carrión, Fernando y Espín, Johanna [comps.], Relaciones fronterizas: Encuentros y conflictos, Ecuador: Facultad Latinoamericana de Ciencias Sociales.

Castañeda, Víctor Mauricio (2015), "La moral tributaria en América Latina y la corrupción como uno de sus determinantes", en Revista Mexicana de Ciencias Politicas y Sociales, año LX, núm. 224, México: Universidad Nacional Autónoma de México. DOI: 10.1016/S0185-1918(15)30005-2 Disponible en: https://doi.org/10.1016/S01851918(15)30005-2 [10 de agosto de 2017].

Coleman, James (1994), Foundations of Social Theory, Estados Unidos: Harvard University Press.

Collins, Randall (1996), Cuatro tradiciones sociológicas, México: Universidad Autónoma Metropolitana.

Daros, William (2005), “Tras las huellas el pacto social”, en Enfoques, vol. XVII, núm. 1, Argentina: Universidad Adventista del Plata Libertador San Martín.

DANE [Departamento Administrativo Nacional de Estadística] (2015), Estimaciones de población 1985-2005 y proyecciones de población 2005-2020 nacional, departamental y municipal por sexo, grupos quinquenales de edad. Disponible en: http://www.dane. gov.co/index.php/poblacion-ydemografia/proyecciones-de-poblacion [31 de julio de 2017].

De Leeuw, J. (2005), “Multivariate analysis with optimal scaling”. Disponible en: http:// repositories.cdlib.org/uclastat/papers/2005103002/ [15 de julio de 2017].

Drobak, John y Nye, John (1997), The Frontiers of the New Institutional Economics, Estados Unidos: Academic Press.

Elster, Jon (1989), "Social Norms and Economic Theory", en Journal of Economic Perspectives, vol. 3, núm. 4, Estados Unidos: Springer Link.

Geiger, P. (1994), "Des-territorialização e espacializacão", en Território: globalização e fragmentaçâo, Brasil: Editora Hucitec.

González, Wilfredo (2016), "Conciencia cívica y ciudadana y prácticas comerciales en el marco del contrabando”, en Albornoz et al., El Táchira en cifras 2015. El Contrabando en las ciudades de frontera desde la perspectiva de la institucionalidad, Venezuela: Universidad Católica del Táchira.

Habermas, Jürgen (1989), The structural transformation of the public sphere: an inquiry into a category of bourgeois society, Estados Unidos: MIT Press.

IBM Knowledge Center (s.f.), Introducción a los procedimientos de escalamiento óptimo de datos categóricos. Disponible en https://www.ibm.com/support/knowledgecenter/ 
Convergencia Revista de Ciencias Sociales, núm. 81, 2019, Universidad Autónoma del Estado de México

es/SSLVMB_22.0.0/com.ibm.spss.statistics.help/spss/categories/categories_ introduction.htm [18 de julio de 2017].

INE [Instituto Nacional de Estadística] (2012), XIV Censo de Población y Vivienda. Población proyectada al 2015. Disponible en http://www.redatam.ine.gob.ve/Censo2011/index. html [18 de julio de 2017].

Mixon, Franklin et al. (2005), "The decay of norms and the production of social order: conceptual and empirical models", en Applied Economics Letters, vol. 12, núm. 1, Reino Unido: Taylor \& Francis online. DOI: 10.1080/1350485042000249797 Disponible en: https://doi.org/10.1080/1350485042000249797 [30 de agosto de 2017].

Morrow, Paul (2014), "Mass Atrocity and Manipulation of Social Norms", en Social Theory and Practice, vol. 40, núm. 2, Estados Unidos: JSTOR. DOI: 10.5840/soctheorpract201440216 Disponible en: https://doi.org/10.5840/ soctheorpract201440216 [28 de agosto de 2017].

Niño, Elizabeth et al. (2012), "Frontera Norte de Santander-Táchira”, en La frontera caliente entre Colombia y Venezuela, Colombia: Nomos Impresores.

North, Douglas (1994), Economic performance through time. Prepared for presentation as the Prize Lecture in Economic Science in Memory of Alfred Nobel, Estados Unidos: Washington University.

Öberg, Perola et al. (2011), "Disrupted Exchange and Declining Corporatism: Government Authority and Interest Group Capability in Scandinavia", en Government and Opposition, vol. 46, núm. 3, Estados Unidos: Cambridge University Press. DOI: 10.1111/j.1477-7053.2011.01343.x. Disponible en: https://doi.org/10.1111/j.14777053.2011.01343.x [23 de septiembre de 2017].

Oddone, Nahuel et al. (2016), "Pactos territoriales en la construcción de regiones transfronterizas: por una mayor integración a múltiples niveles”, en Serie Desarrollo Territorial, Chile: Comisión Económica para América Latina y el Caribe y Naciones Unidas.

Parsons, Talcott (1977), "The Present Status of 'Structural-Functional' Theory”, en Parsons T., Social Systems and The Evolution of Action Theory, Estados Unidos: The Free Press.

Plancarte, Rafael (2015), “¿Son racionales las normas sociales?”, en Espiral, vol. XXII, núm. 64, México: Universidad de Guadalajara.

Posada, Gregorio et al. (2015), "La inobservancia a las normas sociales: hendiduras a la ontología de la realidad social”, en Anagramas, vol. 14, núm. 27, Colombia: Universidad de Medellín.

Prats, Joan Oriol (2007), "Revisión crítica de los aportes del institucionalismo a la teoría y práctica del desarrollo”, en Revista de Economía Institucional, vol. 9, núm. 16, Colombia: Universidad del Externado.

Quijada, Mónica (2000), “Nación y territorio: la dimensión simbólica del espacio en la construcción nacional argentina. Siglo XIX”, en Revista de Indias, vol. 60, núm. 219, España: Consejo Superior de Investigaciones Científicas.

Preisser, John y Koch, Gary (1997), "Categorical data analysis in public health", en Annual Review of Public Health, vol. 18, núm. 1, Estados Unidos: Annual Reviews.

Raffestin, Claude (1986), “Territorialité: concept ou paradigme de la géographie sociale?”, en Geographica Helvetica, vol. 41, núm. 2, Suiza: Copernicus Publication.

Rhi-Sausi, José Luis et al. (2009), "Cooperación e integración transfronteriza en América Latina y el Mercosur”, en Cooperación transfronteriza e integración en América Latina: 
la experiencia del proyecto Fronteras Abiertas, Argentina: Centro Superior para el Procesamiento de Información, Universidad Nacional de la Plata.

Rodríguez-Albor, Gustavo et al. (2016), "Papel de las instituciones en los territorios subnacionales”, en Convergencia. Revista de Ciencias Sociales, vol. 23, núm. 72, México: Universidad Autónoma del Estado de México.

Sack, Robert David (1997), Homo Geographicus. A Framework for Action, Awareness and Moral Concern, Estados Unidos y Reino Unido: The Johns Hopkins University Press.

Sánchez, Luis (2015), "De territorios, límites, bordes y fronteras: una conceptualización para abordar conflictos sociales", en Revista de Estudios Sociales, núm. 53, Colombia: Universidad de Los Andes. DOI: 10.7440/res53.2015.14 Disponible en: http:// dx.doi.org/10.7440/res53.2015.14 [20 de julio de 2017].

Simmel, Georg (1977), "El espacio y la sociedad”, en Estudios sobre las formas de socialización, vol. 2, España: Alianza.

Stein, Eduardo et al. (2015), "Contrabando y Defraudación Aduanera en Centroamérica”, en Documento de Política, núm. 5, Guatemala: Konrad Adenauer Stiftung y Red Centroamericana de Centros de Pensamiento e Incidencia. Disponible en: http:// www.kas.de/wf/doc/kas_41378-1522-4-30.pdf?150520193701 [18 de septiembre de 2017].

Taylor, Hansen y Douglas, Lawrence (2007), "El concepto histórico de frontera”, en Antropología de las fronteras: Alteridad, historia e identidad más allá de la linea, México: El Colegio de la Frontera Norte.

Tejedor, Joan Miguel (2012), "El modelo económico y su relación con los derechos humanos en Colombia. Una aproximación”, en Finanzas y Política Económica, vol. 4, núm. 1, Colombia: Universidad Católica de Colombia.

Traxler, Christian y Spichtig, Mathias (2011), "Social norms and the indirect evolution of conditional cooperation”, en Journal of Economics, vol. 102, núm. 3, Austria: Springer Vienna. DOI: 10.1007/s00712-010-0173-9 Disponible en: https://link.springer. com/content/pdf/10.1007/s00712-010-0173-9.pdf [18 de septiembre de 2017].

Van Der Kooij, Anita (2007), Prediction accuracy and stability of regression with optimal scaling transformations, Holanda: Leiden University. Disponible en: https:// openaccess.leidenuniv.nl/handle/1887/12096 [15 de julio de 2017].

Vargas, Gilbert (2012), "Espacio y territorio en el análisis geográfico”, en Reflexiones, vol. 91, núm. 1, Costa Rica: Universidad de Costa Rica.

Villoria, Manuel y Jiménez, Fernando (2012), “¿Cuánta corrupción hay en España? Los problemas metodológicos de la medición de corrupción (2004-2011)”, en Revista de Estudios Politicos, núm. 156, España: Centro de Estudios Políticos y Constitucionales. Zorrilla, Rubén (1992), Principios y leyes de la Sociología, Argentina: Emecé. 


\section{Anexo}

\section{Tabla 1}

Características de la muestra, error estándar e intervalos de confianza

\begin{tabular}{|c|c|c|c|c|c|c|}
\hline & & $\mathrm{N}$ & $\%$ & $\begin{array}{c}\text { Errores parciales } \\
\text { estándar de la es- } \\
\text { tratificación por } \\
\text { zona geográfica } \\
(\%)\end{array}$ & $\begin{array}{l}\text { Límite } \\
\text { inferior } \\
\text { IC (\%) }\end{array}$ & $\begin{array}{l}\text { Límite } \\
\text { superior } \\
\text { IC }(\%)\end{array}$ \\
\hline \multirow{7}{*}{$\begin{array}{l}\text { Departamento } \\
\text { Norte de } \\
\text { Santander }\end{array}$} & Centro & 69 & 2.9 & 0.34 & 2.3 & 3.6 \\
\hline & Norte & 118 & 4.9 & 0.44 & 4.1 & 5.9 \\
\hline & Occidente & 404 & 16.9 & 0.77 & 15.4 & 18.4 \\
\hline & Oriente & 1.550 & 64.7 & 0.98 & 62.8 & 66.6 \\
\hline & Suroccidente & 149 & 6.2 & 0.49 & 5.3 & 7.2 \\
\hline & Suroriente & 104 & 4.3 & 0.42 & 3.6 & 5.2 \\
\hline & Total & 2.394 & 100 & & & \\
\hline \multirow{6}{*}{ Estado Táchira } & Metropolitana & 560 & 40.1 & 1.31 & 37.5 & 42.6 \\
\hline & Montaña & 147 & 10.5 & 0.82 & 9 & 12.2 \\
\hline & Frontera & 382 & 27.3 & 1.19 & 25 & 29.7 \\
\hline & Norte & 150 & 10.7 & 0.83 & 9.2 & 12.4 \\
\hline & Sur & 159 & 11.4 & 0.85 & 9.8 & 13.1 \\
\hline & Total & 1.398 & 100 & & & \\
\hline
\end{tabular}

Fuente: Elaboración propia. Error estándar e intervalos de confianza 95\%. La estratificación del Departamento Norte de Santander y Estado Táchira se realizó considerando las características físico-espaciales, socioeconómicas y políticas, las cuales definen el funcionamiento espacial de cada uno de sus municipios. 


\section{Tabla 2}

\section{Coeficientes transformados del modelo}

\begin{tabular}{|c|c|c|c|c|c|}
\hline & \multicolumn{2}{|c|}{$\begin{array}{l}\text { Standardized } \\
\text { Coefficients }\end{array}$} & \multirow{2}{*}{$\mathrm{df}$} & \multirow{2}{*}{$\mathrm{F}$} & \multirow{2}{*}{ Sig. } \\
\hline & Beta & Std. Error & & & \\
\hline Nivel educativo & -0.035 & 0.015 & 2 & 5.29 & 0.005 \\
\hline Condición de ocupación & -0.039 & 0.016 & 5 & 6.26 & 0.000 \\
\hline $\begin{array}{l}\text { Existen relaciones de cooperación } \\
\text { entre los funcionarios de seguridad } \\
\text { y los practicantes del contrabando. }\end{array}$ & 0.112 & 0.024 & 2 & 22.75 & 0.000 \\
\hline $\begin{array}{l}\text { Los grupos al margen de la ley son } \\
\text { parte de la dinámica fronteriza. }\end{array}$ & 0.360 & 0.029 & 1 & 151.67 & 0.000 \\
\hline $\begin{array}{l}\text { Grandes cantidades de mercancía } \\
\text { de contrabando es gracias a las re- } \\
\text { laciones de cooperación con los } \\
\text { funcionarios y los comerciantes in- } \\
\text { formales. }\end{array}$ & 0.071 & 0.024 & 1 & 8.37 & 0.004 \\
\hline $\begin{array}{l}\text { Se percibe un ambiente de ilega- } \\
\text { lidad y el comportamiento de las } \\
\text { personas no se basa en normas en la } \\
\text { frontera. }\end{array}$ & 0.283 & 0.028 & 2 & 100.94 & 0.000 \\
\hline $\begin{array}{l}\text { El gobierno nacional y regional ha } \\
\text { notificado y publicado la informa- } \\
\text { ción acerca de los hallazgos, leyes, } \\
\text { decretos y resoluciones relacionadas } \\
\text { al contrabando. }\end{array}$ & -0.030 & 0.015 & 2 & 4.03 & 0.018 \\
\hline
\end{tabular}




\begin{tabular}{|c|c|c|c|c|c|}
\hline & \multicolumn{2}{|c|}{$\begin{array}{l}\text { Standardized } \\
\text { Coefficients }\end{array}$} & \multirow{2}{*}{$\mathrm{df}$} & \multirow{2}{*}{$\mathrm{F}$} & \multirow{2}{*}{ Sig. } \\
\hline & Beta & Std. Error & & & \\
\hline Nivel educativo & 0.033 & 0.018 & 2 & 3.41 & 0.033 \\
\hline Condición de ocupación & 0.022 & 0.014 & 5 & 2.51 & 0.029 \\
\hline $\begin{array}{l}\text { La burocracia en los puestos de se- } \\
\text { guridad para controlar la mercancía } \\
\text { de contrabando ha aumentado el } \\
\text { pago de sobornos. }\end{array}$ & -0.041 & 0.017 & 2 & 5.72 & 0.003 \\
\hline $\begin{array}{l}\text { El Estado Venezolano actúa de ma- } \\
\text { nera eficaz en el control de la mer- } \\
\text { cancía de contrabando. }\end{array}$ & 0.047 & 0.020 & 1 & 5.58 & 0.018 \\
\hline $\begin{array}{l}\text { Los grupos al margen de la ley son } \\
\text { parte de la dinámica fronteriza. }\end{array}$ & 0.482 & 0.029 & 1 & 283.15 & 0.000 \\
\hline $\begin{array}{l}\text { Grandes cantidades de mercancía } \\
\text { de contrabando es gracias a las rela- } \\
\text { ciones de cooperación con los fun- } \\
\text { cionarios y los comerciantes ilegales. }\end{array}$ & 0.053 & 0.020 & 3 & 6.75 & 0.000 \\
\hline $\begin{array}{l}\text { Se percibe un ambiente de ilega- } \\
\text { lidad y el comportamiento de las } \\
\text { personas no se basa en normas en la } \\
\text { frontera. }\end{array}$ & 0.320 & 0.028 & 1 & 126.94 & 0.000 \\
\hline
\end{tabular}

Fuente: Elaboración propia. Variable dependiente: se visualizan pactos sociales informales (acuerdos entre personas y/o instituciones) en la zona de la frontera. 
Neida Albornoz-Arias, Rina Mazuera-Arias, Mª Genoveva Millán-Vázquez de la Torre y Roberto Briceño-León. Los pactos sociales y el contrabando en la frontera colombo-venezolana

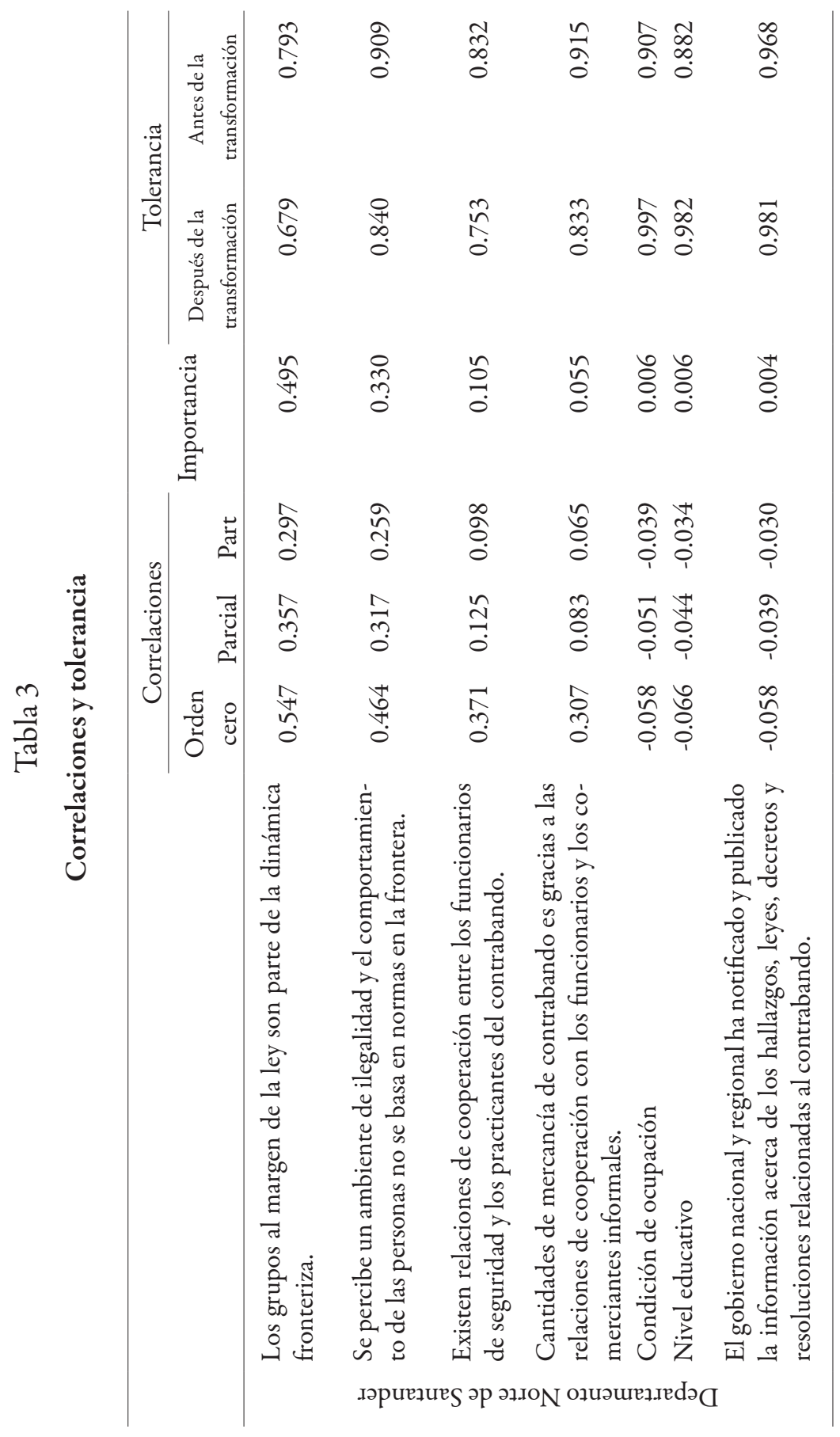




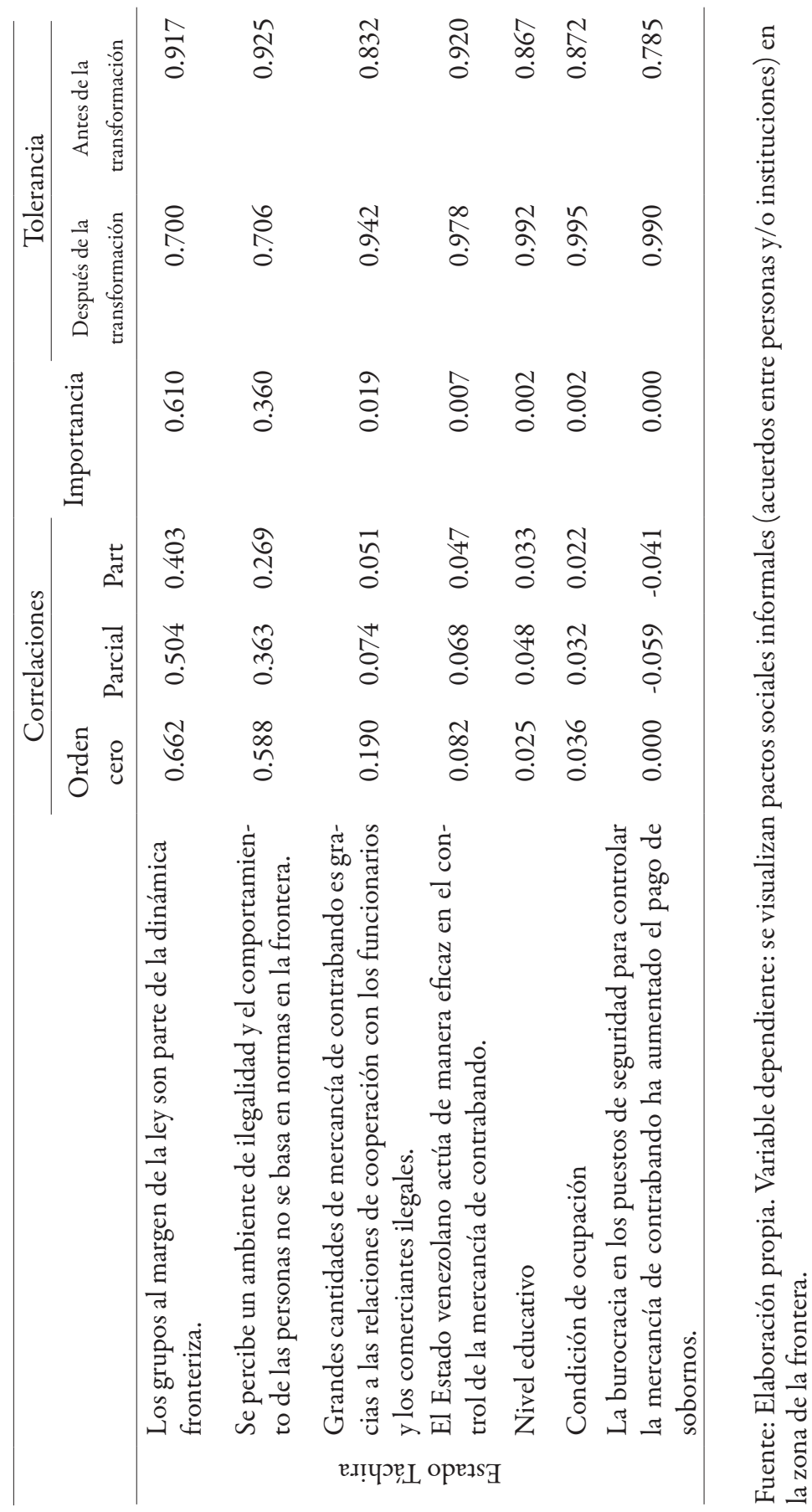


Neida Coromoto Albornoz-Arias. Doctora en Ciencias Sociales y Jurídicas por la Universidad de Córdoba, España. Universidad Simón Bolívar, Colombia, Facultad de Administración y Negocios. Integrante del Grupo de investigación Altos Estudios de Frontera (ALEF), Universidad Simón Bolívar, Cúcuta, Colombia, y del Observatorio Social del Estado Táchira (OSET), Universidad Católica del Táchira, San Cristóbal, Venezuela. Líneas de investigación: estudios socioeconómicos, sociojurídicos y culturales de la frontera colombo- venezolana, cultura de paz. Publicaciones recientes: Albornoz Arias, Neida Coromoto y Mazuera Arias, Rina (2016), “Percepción de habitantes del Norte de Santander sobre limitaciones del buen gobierno", en Revista Venezolana de Gerencia, vol. 21, Venezuela: Universidad Del Zulia; Albornoz Arias, Neida Coromoto (2016), "Contrabando y conflictividad social en la frontera Táchira Norte de Santander", en Aldea Mundo, vol. 42, Venezuela: Universidad de Los Andes; Albornoz Arias, Neida Coromoto et al. (2016), Análisis del contrabando en el Norte de Santander, desde la percepción de los constructos de la institucionalidad, Colombia: Ediciones Universidad Simón Bolívar. Disponible en http://bonga.unisimon.edu.co/ handle/123456789/1146

Rina Mazuera-Arias. Doctora en Derecho por la Universidad de Zaragoza, España. Universidad Simón Bolívar, Colombia. Facultad de Derecho. Integrante del Grupo de investigación Altos Estudios de Frontera (ALEF), Universidad Simón Bolívar, Cúcuta, Colombia, y del Observatorio Social del Estado Táchira (OSET), Universidad Católica del Táchira, San Cristóbal, Venezuela. Líneas de investigación: estudios socioeconómicos, sociojurídicos y culturales de la frontera colombo-venezolana, cultura de paz, familia y sociedad. Publicaciones recientes: Albornoz Arias, Neida Coromoto y Mazuera Arias, Rina (2016), "Percepción de habitantes del Norte de Santander sobre limitaciones del buen gobierno", en Revista Venezolana de Gerencia, vol. 21, Venezuela: Universidad Del Zulia; Albornoz Arias, Neida Coromoto et al. (2016), Análisis del contrabando en el Norte de Santander, desde la percepción de los constructos de la institucionalidad, Colombia: Ediciones Universidad Simón Bolívar. Disponible en: http://bonga.unisimon.edu.co/ handle/123456789/1146. 3); Alba Niño, Magaly et al. (2016), Percepción de los habitantes de las subregiones del Norte de Santander frente al contrabando, Colombia: Ediciones Universidad Simón Bolívar. Disponible en: http:// bonga.unisimon.edu.co/handle/123456789/1131 


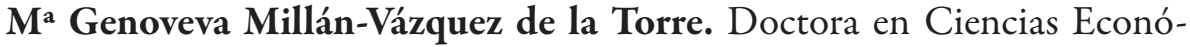
micas y Empresariales, Facultad de Ciencias Económicas y Empresariales, Universidad Loyola Andalucía, España. Líneas de investigación: turismo y empleo. Publicaciones recientes: Millán, M. G., Amador, L. \& Arjona, M. J. (2016), "The Protected Designation of Origin 'Los Pedroches' as a Culinary Route for Iberian Ham: Analysis of the Visitor Profile and Future Evolution”, en Cuadernos de Desarrollo Rural, vol. 13, núm. 77, Colombia: Universidad Javeriana; Millán, M. G., Velasco, M. T. y Ramírez, J. (2017), "El emprendimiento de la mujer rural española: análisis de la brecha salarial. Una realidad difícil de solucionar”, en Papeles de Población, vol. 23, núm. 92, México: Universidad Autónoma del Estado de México; Millán, M. G., Hidalgo, L. A. y Fuentes, J. M. A. (2016), "El paisaje agavero y la planificación turística sostenible. Un requisito para su permanencia como sitio patrimonio de la humanidad", en Scripta Nova. Revista Electrónica de Geografía y Ciencias Sociales, vol. 20, núm. 537, España.

Roberto Briceño-León. Postdoctorado en el Centro Latinoamericano de Saint Antony's College de la Universidad de Oxford, Reino Unido. Universidad Central de Venezuela, Facultad de Ciencias Económicas y Sociales, Escuela de Sociología. Líneas de investigación: sociología de la violencia y sociología de la salud. Publicaciones recientes: Briceño-León, Roberto (2017), “¿Qué enseña el fracaso en la reducción de homicidios en Venezuela?”, en Revista CIDOB d'Afers Internacionals, núm. 116, España: Barcelona Centre for International Affairs; Briceño-León, Roberto (2016), "La sociología de la Violencia: un campo nuevo", en Espacio abierto: Cuaderno Venezolano de Sociología, vol. 25, núm. 4, Venezuela: Universidad del Zulia; Briceño-León, Roberto y Ávila Fuenmayor, Olga (2014), “De la participación comunitaria a la participación social: un enfoque de Ecosalud", en Espacio abierto: Cuaderno Venezolano de Sociología, vol. 23, núm. 2, Venezuela: Universidad del Zulia. 